\title{
A quasi-Delphi study on technological barriers to the uptake of hydrogen as a fuel for transport applications-Production, storage and fuel cell drivetrain considerations
}

\author{
David Hart ${ }^{\mathrm{a}, *}$, Alexandra T. Anghel ${ }^{\mathrm{b}}$, Joep Huijsmans ${ }^{\mathrm{b}}$, François Vuille ${ }^{\mathrm{a}}$ \\ a E4tech Sàrl, Avenue Juste-Olivier 2, 1006 Lausanne, Switzerland \\ b Shell Hydrogen B.V., Carel van Bylandtlaan 23, 2596 HR The Hague, The Netherlands
}

\section{A R T I C L E I N F O}

\section{Article history:}

Received 10 October 2008

Received in revised form 11 December 2008

Accepted 11 December 2008

Available online 14 January 2009

\section{Keywords:}

Fuel cell

Hydrogen

Transport

Barriers

Expert analysis

Delphi

\begin{abstract}
A B S T R A C T
The introduction of hydrogen in transport, particularly using fuel cell vehicles, faces a number of technical and non-technical hurdles. However, their relative importance is unclear, as are the levels of concern accorded them within the expert community conducting research and development within this area. To understand what issues are considered by experts working in the field to have significant potential to slow down or prevent the introduction of hydrogen technology in transport, a study was undertaken, primarily during 2007. Three key technology areas within hydrogen transport were selected - hydrogen storage, fuel cell drivetrains, and small-scale hydrogen production - and interviews with selected experts conducted. Forty-nine experts from 34 organisations within the fuel cell, automotive, industrial gas and other related industries participated, in addition to some key academic and government figures. The survey was conducted in China, Japan, North America and Europe, and analysed using conventional mathematical techniques to provide weighted and averaged rankings of issues viewed as important by the experts. It became clear both from the interviews and the subsequent analysis that while a primary concern in China was fundamental technical performance, in the other regions cost and policy were rated more highly. Although a few individual experts identified possible technical showstoppers, the overall message was that pre-commercial hydrogen fuel cell vehicles could realistically be on the road in tens of thousands within 5 years, and that full commercialisation could take place within 10-15 years, without the need for radical technical breakthroughs. Perhaps surprisingly, the performance of hydrogen storage technologies was not viewed as a showstopper, though cost was seen as a significant challenge. Overall, however, coherent policy development was more frequently identified as a major issue to address.
\end{abstract}

(c) 2009 Elsevier B.V. All rights reserved.

\section{Introduction}

The introduction of fuel cell vehicles and hydrogen fuel into transport faces a range of challenges, both technical and nontechnical [1,2]. Scientific analysis of the challenges and advances in PEM fuel cells has been conducted in depth (e.g. $[3,4])$. However, research into such challenges tends to focus on an individual area, such as the implications of hydrogen storage capacity (e.g. $[5,6])$, and do not give an indication of the general consensus as to their gravity, nor whether they are universally considered in the same light. This study is an initial investigation into the possible variations in the way the different relevant actors picture the challenges ahead. Shell Hydrogen has conducted internal assessments of the technical challenges to hydrogen uptake as a fuel for transport applications in order to identify both showstoppers and any

\footnotetext{
* Corresponding author. Tel.: +41 21331 1579; fax: +41 213311561.

E-mail address: hart@e4tech.com (D. Hart).
}

key areas for Shell engagement. For this analysis, in order to conduct a wider survey, Shell engaged E4tech to interview experts in three key areas to ascertain their views on technology showstoppers or other key issues facing the development/deployment of hydrogen and fuel cell vehicles. These areas are: fuel cell drivetrains, on-board hydrogen storage, and small-scale hydrogen production. Experts from Japan, China, North America and Europe were interviewed to ascertain, as far as possible, differences in concerns and perspectives within these different regions.

The outcomes of this study are reported here in brief and represent E4tech's interpretation of the results as obtained. They do not necessarily in any way reflect Shell's view of these issues. Errors and omissions are due solely to E4tech.

\section{Methodology}

\subsection{Introduction}

The study was based on individual interviews of a sample of independent experts covering a wide range of expertise, insti- 
tutional context and geographic origin. However, overall the sample size was small for the number of topics assessed, hence the make-up of experts in different regions was slightly different. For example, in China, more academics were represented, and Japan had a slightly greater focus on hydrogen storage than did other regions. Although only a single round of interviews was conducted, the interviews were conducted in such a way intended to approach a Delphi methodology. The multiple interview rounds that would typically characterise a Delphi analysis were replaced by a refinement of the questions asked during the course of the interviews, based on responses already obtained:

First, the interviewees were asked to identify issues in their sector of expertise without overt prompting from the interviewer, as it was of prime importance to gather a list of issues as large as possible to start with. Comments around these answers were gathered to ensure the full context and the right perspectives were considered during the subsequent analysis phase.

Then, the interviewer would play the role of the facilitator in the Delphi method by broadening and deepening the discussion around the issues evoked by the interviewee to ask his view on neighbouring issues or root causes identified by previous experts. For instance, if stack cost is an issue, the interviewer would ask what exactly makes the stack expensive (root cause) and prompt if necessary with answers received during past interviews. Views on BoP cost (neighbouring issue) would be solicited to balance with stack costs.

To avoid the last interviews being much more comprehensive than the first, due to a growing set of identified issues, a list of possible issues was initially worked out based on existing knowledge and a small pilot interview survey. This list was refined and extended during the course of the survey. The interviews were originally planned on the understanding that technical issues would form the majority of concerns, and that detail on specific technical sub-issues would be very important. During the process it became evident that other issues, such as policy and deployment strategy, were also of significant interest.

\subsection{The interview template}

An interview template was developed to both structure the interviews and enable the answers received to be recorded in a format suitable for subsequent data analysis. Five specific "issue categories" were defined to ensure that each interview had the same structure and covered the same type of issues:

- technical performance. This includes such characteristics as efficiency, power density etc. and is primarily concerned with scientific or engineering improvements

- dependability. This compound category includes issues related to, e.g. reliability, durability, lifetime, safety

- manufacturing-issues such as process scale-up, redesign of existing components for ease of manufacture, or materials supply

- cost is of course dependent both on intrinsic materials costs and manufacturing complexity

- the 'softer' issues of market deployment were considered, including codes and standards, public acceptance, and policy.

Each expert was asked to rank identified issues according to the following criteria for subsequent analysis and evaluation:

- The severity of an issue-what the implications would be if no solution to a problem were found, ranking from 1 to 3 , with 3 defined as a showstopper.
- The confidence level of the interviewer as to the severity of the issue, ranking from 1 to 3 , with 3 defined as an absolute certainty.

- The chance of solving the problem, rated in percent.

- The solving horizon, i.e. the possible length of time (in years) to a solution

It is clear that many aspects are in fact inter-related, and that cost can be reduced by sacrificing performance or longevity, for example. One of our aims was to ascertain if specific influences were more fundamental. For example, a stack might be designed for high efficiency and lifetime but hence at high cost, and so relaxation of the efficiency and lifetime criteria, once achieved, could lead to lower cost. A stack designed for low cost only might not be able to be redesigned for high efficiency and lifetime.

\subsection{The interview process}

The interview process was open and flexible-the interviewees were given background information as to how the analysis would be conducted. Given the complexity of the subject, the need for consistent interpretation of observations and the requirement for a broad range of understanding of deeply technical, policy and other issues, all of the interviews were conducted by a single interviewer with a strong background in fuel cells and hydrogen.

The questionnaire and interview method were tested on a small sample of experts before launch. These pilot interviews helped the process and questions to be refined and any major methodological problem areas to be identified. It is recognised that neither this sample nor the small sample size of potential interviewees enables the analysis to be shown as statistically robust. However, finding and interviewing a suitably representative sample from all regions, industries and specialisations would not have been possible given time and resource constraints, and so expert judgement was relied upon in many cases for interpreting the answers received and being able to compare those from different interviews.

Forty-nine interviews of experts in China, Japan, North America and Europe were conducted over a period from the end of 2006 until mid-2007. Because of cultural and language differences in the different regions, Chinese and Japanese experts were interviewed face-to-face, while other interviews were conducted by telephone. Thirty-four organisations participated, many with several experts or experts representing different topics. These organisations are listed in Acknowledgements, but under the terms of participation no individual is identified. Experts were approached based on a combination of (a) deep technical understanding and (b) a systems overview. This was to allow the interview to identify not only the specific detail of any technical issues identified, but also the implications for the system as a whole.

Many experts were understandably uncomfortable specifying precise values for uncertain events, such as how long before an issue might be solved, or the chance that a solution would be found. In many cases it was necessary to use our judgement to add a value that we felt reflected the position of the interviewee relative to other interviewees.

\subsection{Data preparation}

Data recorded in the interview template were subsequently transferred to an analysis tool developed within the project. The wide range of issues identified by the experts and their varied technical depth rendered data preparation essential to enable related issues to be compared. For example, one expert might suggest that nitrogen-based compounds in the air could adversely affect cathode catalysts within the fuel cell, while another might identify the fundamental properties of the polymer used within many fuel cells as inherently unsuitable for long-term 


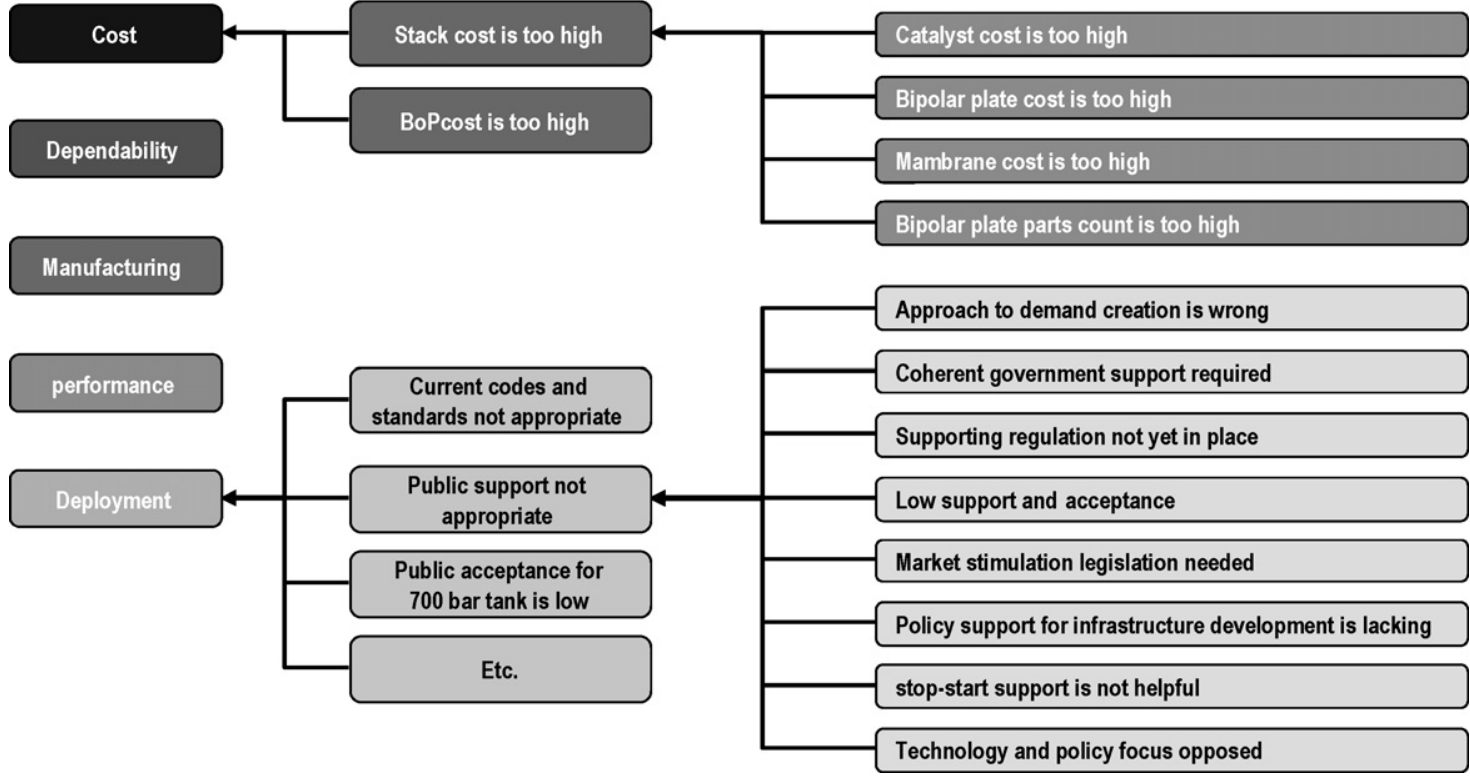

Fig. 1. Extract from the fuel cell drivetrain 3-level tree structure for classification of issues.

stable operation under typical driving conditions, while a third more system-overview type expert might simply identify catalyst lifetime as an issue without knowing about the root cause of the problem. Although these are different issues, at a different depth within the framework, all 3 are related to MEA lifetime.

To enable direct comparison of such issues, a 3-level tree structure starting with the 5 issue categories was developed, a portion of which is shown in Fig. 1. This tree structure was used to classify each issue identified by the experts in a systematic way into predefined categories, enabling subsequent result concatenation and statistical analysis. The 108 issues identified by the experts interviewed were concatenated into 24 generic issues, further classified into the 5 issue categories. A tree structure was developed for the fuel cell drivetrain, hydrogen storage and hydrogen production.

Obviously, judgments had to be made as to appropriate headings for each group of issues. For example, the cost of catalyst and the cost of membrane can be grouped under the cost of MEA, and potentially cost of stack, but cannot be grouped with cost of other components. Inevitably this introduced some subjectivity into the analysis, but it was felt that this was in line with other uncertainties in the analysis.

\subsection{Analysis}

Analysis was carried out using a tool developed to statistically analyse the data collected during the interviews and produce numerical trends and graphical results. It was designed to:

- sort the issues according to all 3 levels of the above tree structure and produce concatenated results tables (see Tables 1 and 2);

- perform simple weighted average calculations on the issues at all 3 levels of concatenation;

- produce a set of graphics of various types (see Section 3).

As a simplifying assumption, issues are considered to be independent of each other. After careful review of the issues identified, this assumption is felt to be reasonable. An algorithm based on Morgan's theorem of probability is used to compute the cumulated probability of independent issues.

It was recognised that due to the limited number of interviews and the wide range of topics, it was not possible to get a statistically significant set of results from the analysis, though some trends emerged. The analysis used a compounding method for several of the issues identified. In addition to the concatenations described

Table 1

Concatenation of the answers at the specific issue level, where each line represents a given issue.

\begin{tabular}{|c|c|c|c|c|c|c|}
\hline Issue category & $\begin{array}{l}\text { Total number of } \\
\text { responses }\end{array}$ & $\begin{array}{l}\text { Weighted number of } \\
\text { responses }\end{array}$ & $\begin{array}{l}\text { Average weighted } \\
\text { severity }\end{array}$ & $\begin{array}{l}\text { Average non-solving } \\
\text { probability (\%) }\end{array}$ & $\begin{array}{l}\text { Average issue } \\
\text { criticality }\end{array}$ & $\begin{array}{l}\text { Average solving } \\
\text { horizon }\end{array}$ \\
\hline Dependability & 5 & 25 & 1.8 & 5 & 0.08 & 2012 \\
\hline Performance & $\begin{array}{l}3 \\
1\end{array}$ & $\begin{array}{r}13 \\
5\end{array}$ & $\begin{array}{l}2.4 \\
2.0\end{array}$ & $\begin{array}{l}17 \\
10\end{array}$ & $\begin{array}{l}0.40 \\
0.20\end{array}$ & $\begin{array}{l}2021 \\
2011\end{array}$ \\
\hline Deployment & $\begin{array}{l}1 \\
1 \\
3 \\
1\end{array}$ & $\begin{array}{r}5 \\
5 \\
13 \\
5\end{array}$ & $\begin{array}{l}2.0 \\
2.0 \\
2.0 \\
2.0\end{array}$ & $\begin{array}{l}10 \\
15 \\
12 \\
25\end{array}$ & $\begin{array}{l}0.20 \\
0.30 \\
0.23 \\
0.50\end{array}$ & $\begin{array}{l}2011 \\
2021 \\
2012 \\
2021\end{array}$ \\
\hline Cost & $\begin{array}{l}1 \\
1 \\
1\end{array}$ & $\begin{array}{l}5 \\
5 \\
5\end{array}$ & $\begin{array}{l}2.0 \\
2.0 \\
2.0\end{array}$ & $\begin{array}{l}5 \\
5 \\
1\end{array}$ & $\begin{array}{l}0.10 \\
0.10 \\
0.02\end{array}$ & $\begin{array}{l}2016 \\
2011 \\
2011\end{array}$ \\
\hline
\end{tabular}


Table 2

Concatenation of all the issues identified, arranged by issue category.

\begin{tabular}{|c|c|c|c|c|c|c|}
\hline Issue category & $\begin{array}{l}\text { Number of } \\
\text { issues }\end{array}$ & $\begin{array}{l}\text { Category average } \\
\text { severity }\end{array}$ & $\begin{array}{l}\text { Category } \\
\text { cumulated severity }\end{array}$ & $\begin{array}{l}\text { Category average } \\
\text { non-solving probability (\%) }\end{array}$ & $\begin{array}{l}\text { Cumulated non-solving } \\
\text { probability (\%) }\end{array}$ & Category criticality \\
\hline Dependability & 1 & 1.8 & 1.8 & 5 & 5 & 0.1 \\
\hline Performance & 2 & 2.2 & 4.4 & 13 & 25 & 0.5 \\
\hline Manufacturability & 0 & 0.0 & 0.0 & 0 & 0 & 0.0 \\
\hline Deployment & 4 & 2.0 & 8.0 & 15 & 49 & 1.0 \\
\hline Cost & 3 & 2.0 & 6.0 & 4 & 11 & 0.2 \\
\hline
\end{tabular}

below, a measure of 'criticality' was introduced, relating the probability of not solving an issue and its severity. An issue that was considered very hard to solve but with an impact that was not very severe would rank as less 'critical' than one which was both hard to solve and had a severe impact.

To investigate some of the sensitivity behind the analysis, different weighting factors were used for the expert's level of confidence. This factor had negligible influence on the results, as the vast majority of experts interviewed claimed to be fully confident about the issues they had identified.

\section{Results}

Results of the interview analysis are grouped in different ways, by geographic region and for the 'world' as a whole. Figures illustrating results have the probability of not solving a particular issue shown on the $y$-axis. This may be given as a raw value (identified by the expert), or a cumulated value (e.g. if a probability exists that the catalyst cost will remain too high, and a probability that the membrane cost will remain too high, then there is a cumulated probability that the cost of the MEA will remain too high, calculated from the two original probabilities).

The $x$-axis of these charts represents either the severity of the impact if the issue is not solved, or the horizon over which it is estimated that it will be solved. Again, these may be raw or averaged, the latter only relevant where multiple experts select the same issue. Bubble size varies, representing either the number of experts identifying a particular issue, in the case where severity is not already shown, or the severity of the issue. Where all bubbles are equal, the size has no meaning.

\subsection{World}

\subsubsection{Issues}

A perspective on the range of issues identified by different experts globally can be seen in Fig. 2, where a first stage concatenation has been undertaken-i.e. very specific issues are grouped into areas of close similarity. An example might be the grouping of 'air compressor cost is too high' and 'heat exchanger cost is too high' under 'BoP cost is too high'. Many different concerns still exist and the chart is somewhat difficult to interpret.

Concatenating the issues further gives more clarity, as shown in Fig. 3, where generic areas and levels of concern can be identified, at the expense of detail.

High pressure tank cost can be seen to concern many experts globally, and solving this issue is felt to have something like a 75\% probability. Dependability of hydrogen production plant has the highest severity factor, though this is clearly of concern to fewer experts. Stack cost being too high, and fuel cell system dependability being too low, were the issues identified by most experts, though inappropriate codes and standards also figured highly, along with hydrogen storage density concerns.

Viewing the same issues by time to solution as in Fig. 4 shows a different spread, with the problem of competing against strong
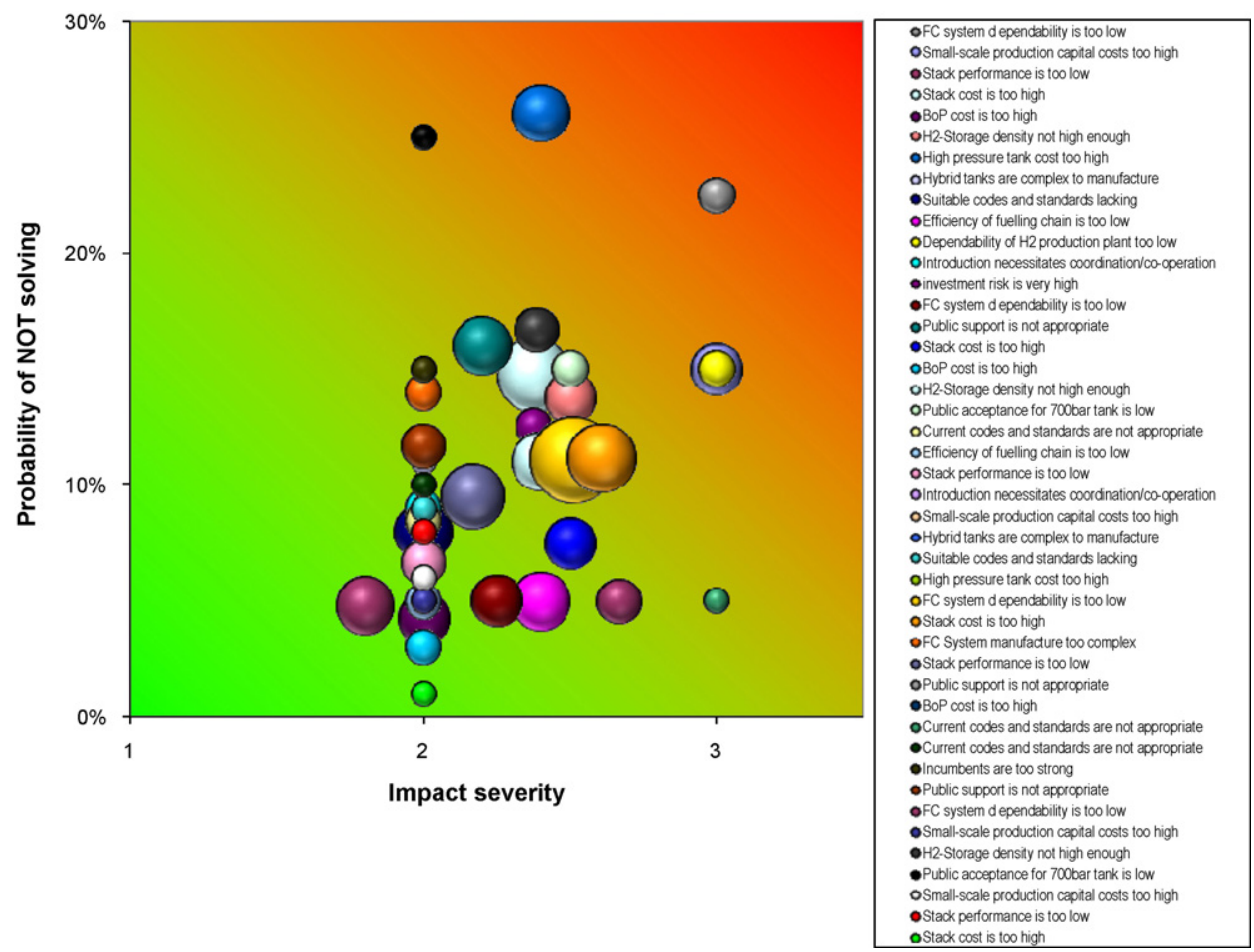

Fig. 2. Views on technical issues globally by probability and severity (pre-concatenated). 


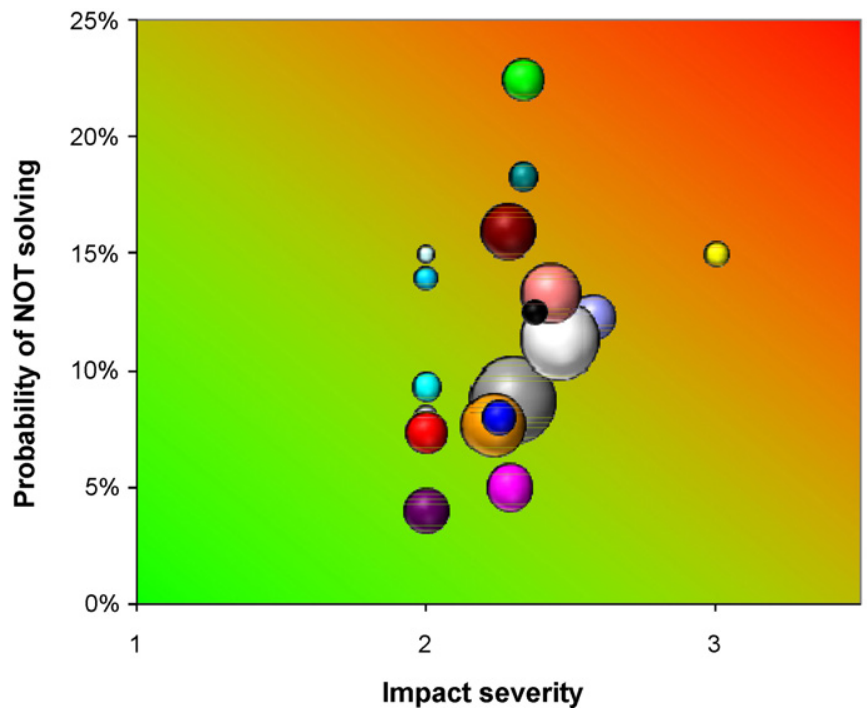
OFC system dependability is too low
oSmall-scale production capital costs too high
OStack performance is too low
QStack cost is too high
BoP cost is too high
oH2-Storage density not high enough
- High pressure tank cost too high
OHybrid tanks are complex to manufacture
- Suitable codes and standards lacking
-Efficiency of fuelling chain is too low
ODependability of $\mathrm{H} 2$ production plant too low
OIntroduction necessitates coordination/co-operation
- investment risk is very high
- Public support is not appropriate
- Public acceptance for 700 bar tank is low
- Current codes and standards are not appropriate
oFC System manufacture too comple $x$
Olncumbents are too strong

Fig. 3. Views on technical issues globally by probability and severity (fully concatenated).

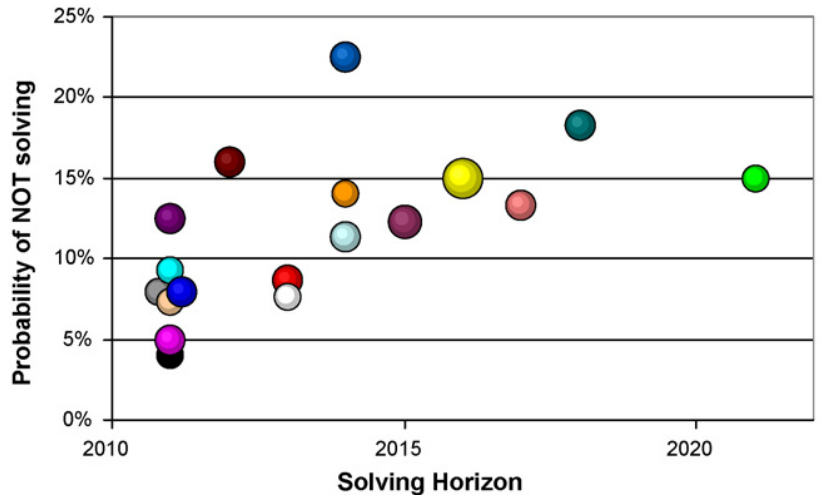

FC system dependability is too low

- Small-scale production capital costs too high

O Stack performance is too low

O Stack cost is too high

- BoP cost is too high

O H2-Storage density not high enough

- High pressure tank cost too high

- Hybrid tanks are complex to manufacture

- Suitable codes and standards lacking

o Efficiency of fuelling chain is too low

O Dependability of $\mathrm{H} 2$ production plant too low

O Introduction necessitates coordination/co-operation

- investment risk is very high

- Public support is not appropriate

- Public acceptance for 700 bar tank is low

- Current codes and standards are not appropriate

O FC System manufacture too complex

O Incumbents are too strong

Fig. 4. Views on technical issues globally by probability and horizon to solution (fully concatenated).

incumbents identified as taking the longest time to solve. Achieving suitable hydrogen storage density and the public acceptance of high pressure tanks were viewed as requiring around 10 years, while small-scale production plant capital costs and dependability were close behind. Interestingly, fuel cell drivetrain issues typically appear in the nearest timeframe to solution, with cost issues slightly more extended.

Concatenating the different issues into the original categories, as shown in Fig. 5, dependability is shown to have the highest severity, with all other issues clustered together. No issue area ranks close to 3-the showstopper category. This corresponds to some of the experts' views that corporate technology roadmaps for commercialisation were solid and well-founded.

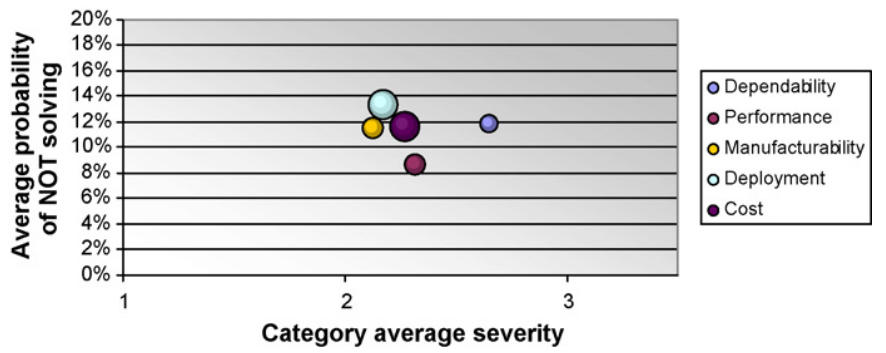

Fig. 5. Views on technical issues globally by probability and average severity, shown by issue category.
Fig. 6 shows the same results ranked by 'criticality'. The numbers along the $y$-axis only serve to position issues relative to each other. Cumulated criticality is the sum of the probabilities of not solving the issues within a particular category. Deployment and cost are clearly the primary concerns for future introduction of hydrogen fuel cell transport, with issues such as public support, under deployment, attracting significant concern. This contrasts strongly with the issue category that was the original subject for

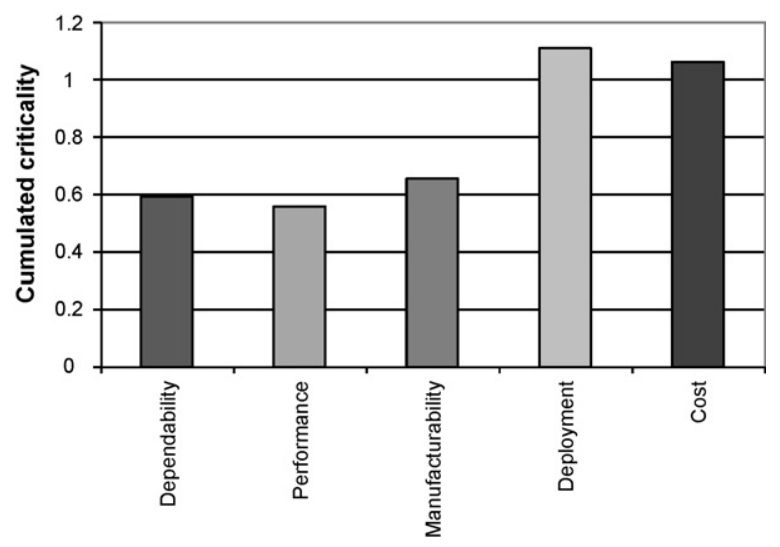

Fig. 6. Views on global cumulated criticality by issue category. 


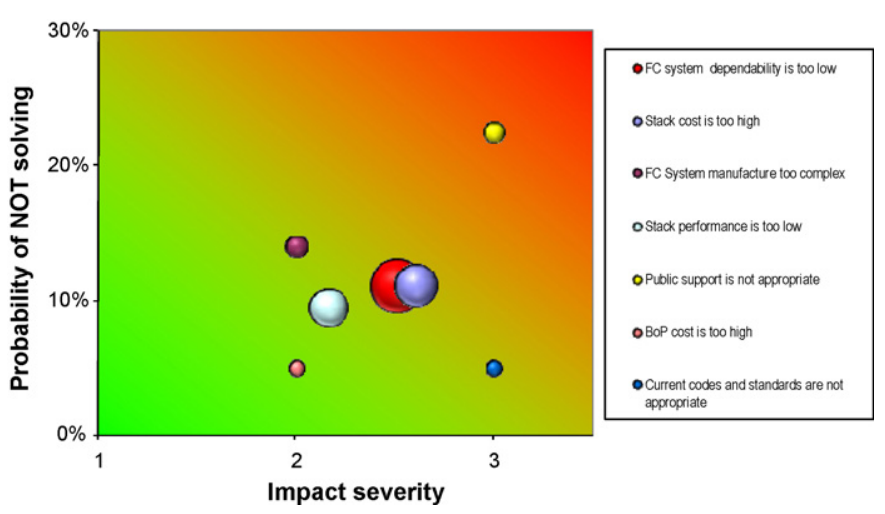

Fig. 7. Expert views on technology issues by severity and improbability of solution (China).

this study-technical performance. This ranks lowest in criticality of all the issue categories, though by a small margin.

It appears from the results above that considerable progress has been made with respect to technical performance of fuel cell drivetrains and associated equipment. A range of experts worldwide has identified pathways that they anticipate will allow fuel cell drivetrains to be technically competitive with many conventional systems within a 5-10 year timeframe. However, the cost of these systems is a slightly higher concern, and their actual deployment, relying on the development of many new supply chains and significant co-ordination between a range of different actors, is an even higher concern.

It was repeatedly emphasised that individual technical problems - catalyst loss, membrane degradation due to hydrogen peroxide formation, contaminant resistance etc. - could each be solved using known techniques. However, many experts emphasised that the major complexity arose from the interaction between issues.

\subsection{Comparing and contrasting regional views}

Results from the different regions showed noticeable differences between expert views, though more similarities were apparent between North America and Europe than any other regions.

\subsubsection{China}

In China almost no research and development of small-scale hydrogen production is underway, and also limited work on onboard storage except at a very fundamental level. Interviews in China were thus strongly focused on fuel cell drivetrains. The interviews charted in Fig. 7 showed that by far the main concern in China was fuel cell durability, though views on the fundamental causes differed. However, membrane and catalyst were both identified as key areas for durability improvement.

Public acceptance was rated more highly, but by only one expert, and is hence unrepresentative of overall opinion. Many Chinese experts commented that high temperature membranes would be helpful in overcoming a range of problems, from heat rejection in the vehicle through contaminant resistance. Improving materials performance and system performance as a route to reducing cost is generally accepted, rather than focusing on cost reduction first.

Cost, although identified as important, was much less a concern than durability. Many felt that China had the competence and potential to produce most fuel cell materials at low cost, with the possible exception of catalyst. Individual Chinese provinces have support programmes for key industries, such as membrane production, and these could provide platforms for fuel cell materials. Because platinum is a global commodity, China cannot influence its cost other than through the market, and so work is being conducted

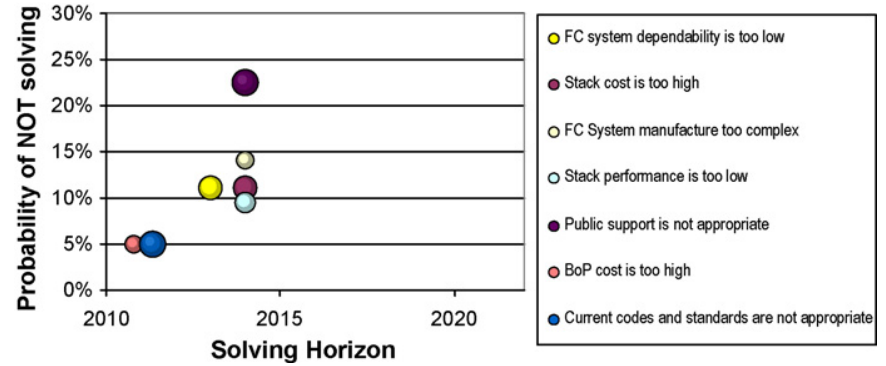

Fig. 8. Expert views on technology issues by solving horizon and improbability of solution (China).

on replacements. However, scaling up any eventual alternative catalyst production could be a major issue, as many catalyst samples for fuel cells are only made in grams.

One inference that can be drawn from the interviews is that of technology development level. China began investing significant amounts into fuel cell systems more recently than many of the other regions, and has fewer major corporations with demonstration experience. Despite rapid advances, it is possible that Chinese scientists and engineers are behind other regions in terms of solving some key technical problems. This is reinforced by the detailed comments raised in interviews, where problems of catalyst sintering and dissolution, membrane microstructure and similar issues are mentioned. While these problems remain intractable globally, systems engineering in other regions appears to have advanced to a point where they are viewed as less of a fundamental problem.

Chinese experts are, however, comparatively optimistic on solving horizons-as shown in Fig. 8. Each issue identified was considered to be solvable within 10 years.

Concatenating the issues, as in Fig. 9, shows that cost and deployment dominate, though cost is not considered to be as severe an issue. In contrast, deployment of fuel cell vehicles in China is viewed as a very severe problem, due to a perceived lack of government initiative. While the national government has strongly supported fuel cell research and development, limited funds are being put into deployment. Given the wide range of competing near term alternatives to fuel cells, including DME, methanol and biofuels, many experts suggest that China is not likely to have widespread deployment of fuel cell vehicles in the near future. An additional hurdle to deployment is infrastructure. Public funding is required along with co-ordination, and little has been identified at this stage. Some Chinese standards make it difficult to construct stations, and less funding is being put into infrastructure than other areas. A further complication is the extremely limited number of organisations in China investigating hydrogen production methods or refuelling infrastructure.

Acceptance of hydrogen technologies by the general public or other groups was not felt to be an issue by the majority of people.

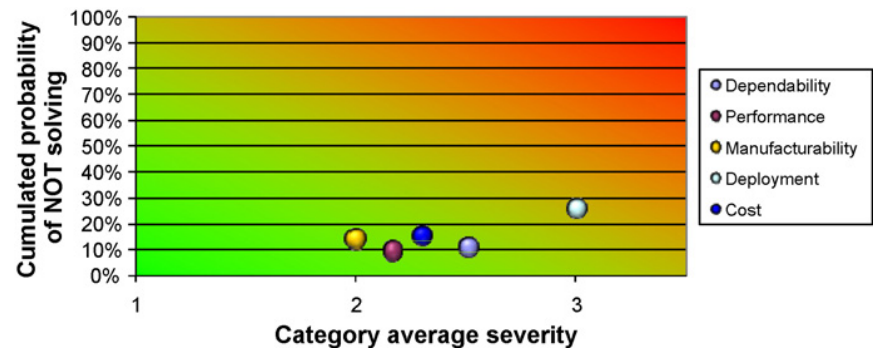

Fig. 9. Views on technology issues by severity and improbability of solution at issue category level (China). 


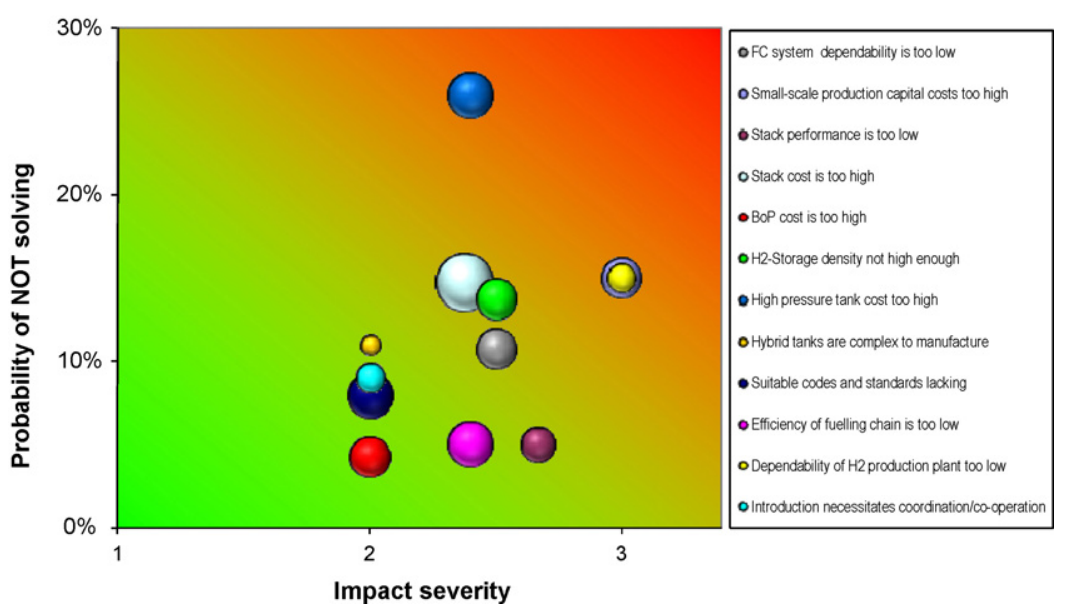

Fig. 10. Expert views on technology issues by severity and improbability of solution (Japan).

\subsubsection{Japan}

Japanese experts identified a range of issues of concern, as shown in Fig. 10, though small-scale hydrogen production plant dependability and cost were seen as particularly troubling. This is understandable once Japan's energy situation is considered, with all transport fuel imported along with the vast majority of all fuels. Production of hydrogen requires a raw resource which is imported (e.g. natural gas in the form of LNG) and is hence already expensive. Conversion into hydrogen must therefore add little cost, so capital costs, dependability and efficiency of hydrogen production plants are critical.

The technology issues identified were varied, with interesting contrasts. OEMs consistently claimed to have pathways to development of technically competitive fuel cell solutions, while researchers were less positive, a theme repeated in North America. Very specific and fundamental technical issues were cited, including:

- poor understanding of the oxygen reduction reaction on the cathode, which results in much higher catalyst loadings than theoretically required and may be linked to the unsuitability of the carbon support;

- poor understanding of interactions at the membrane/ionomer/catalyst/GDL interface limits the development of solutions to problems of poor catalyst utilisation and specific types of degradation. Equally, hydrogen peroxide formation mechanisms - from molecules to clusters to the final chemical - as the spatial resolution of existing equipment is too low. Breakthroughs in understanding in the former could lead to radical steps such as GDL-free fuel cells.

- simpler polymers are ultimately required, as fluorine-based polymers are inherently too expensive.

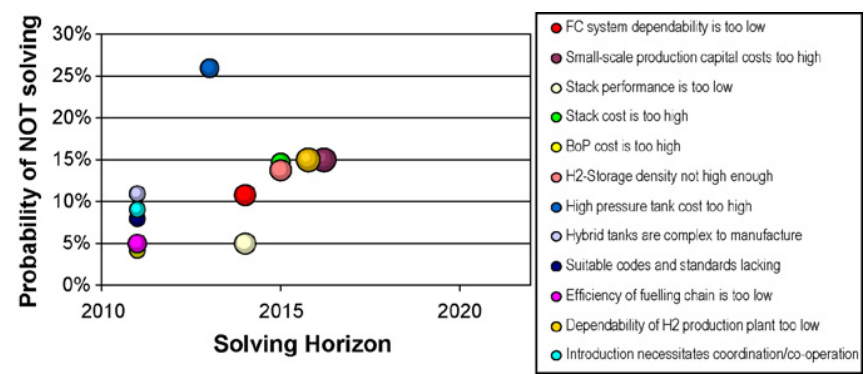

Fig. 11. Expert views on technology issues by solving horizon and improbability of solution (Japan)
Stack and systems cost was a lesser concern, having a lower severity than some other issues, though it was frequently cited and has a comparatively high possibility of not being solved.

For on-board storage of hydrogen two contrasting sets of opinions were strongly presented. High volumetric energy density with a flexible form factor was considered the most important target by some, on the grounds that weight could be managed within the vehicle in other ways but that bulk was problematic to deal with. Others expressed exactly the opposite view, stating that weight was the most important contributor to a vehicle's performance and that volume could be distributed around the vehicle, leading to a focus on gravimetric energy density. Although these different philosophies are not easily reconciled, general agreement was that current high pressure technology is adequate for the first phase of vehicle introductions, and that hydrogen storage is not a key barrier. Common balance of plant with some existing stationary systems may enable crossover learning and development between the two areas. A less severe issue but with higher probability of not being solved was high pressure tank cost. While experts almost universally agreed that high pressure hydrogen would be the near- and mid-term storage solution, many cited the high cost of suitable grades of carbon fibre, responsible for $\sim 75 \%$ of the final tank cost, as a major issue. High aerospace industry demand for carbon fibre and a limited supply base combine to keep prices very high, with few near-term possibilities for this to change. That this issue figured more highly than some anticipated issues, e.g. catalyst cost, may be viewed as somewhat surprising. It could be due to the make-up of the expert interviewees, or possibly to recent activities in high pressure storage which meant that it was at the forefront of people's minds.

As shown in Fig. 11, Japanese researchers considered that most issues could be solved in a timeframe between 5 and 10 years, with stack and balance of plant cost identified as only becoming

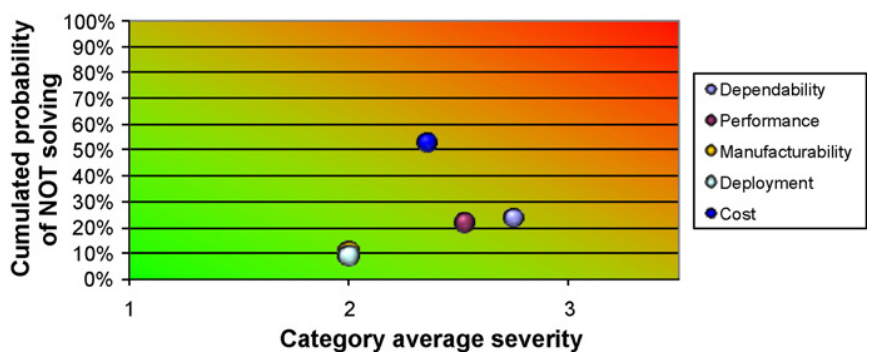

Fig. 12. Views on technology issues by severity and improbability of solution at issue category level (Japan) 


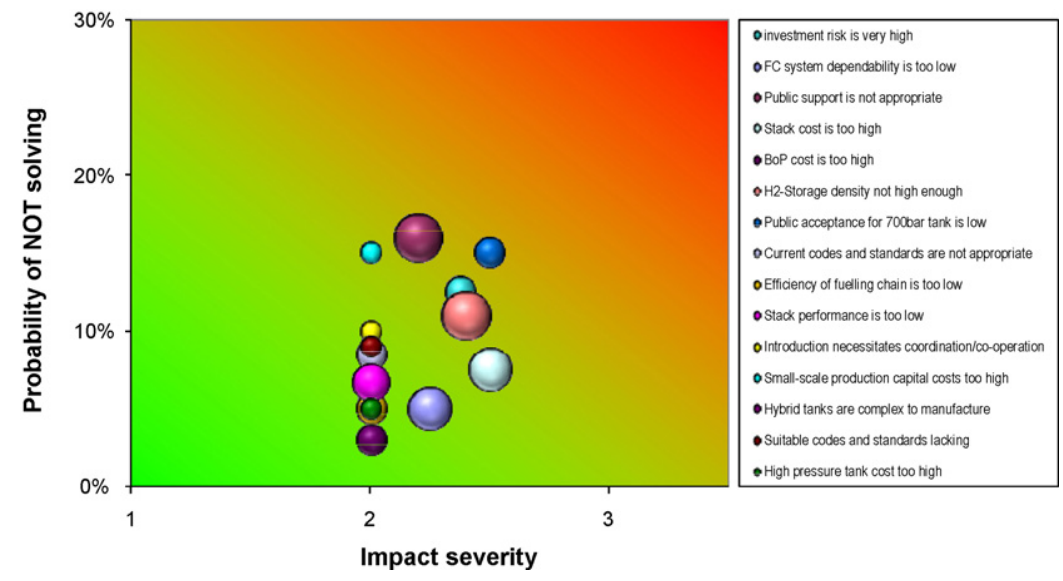

Fig. 13. Expert views on technology issues by severity and improbability of solution (North America).

fully competitive towards the end of that timeframe, in addition to hydrogen storage density.

Although dependability was viewed as the most severe issue of the different types (see Fig. 12), relatively few experts identified it and it was viewed as likely to be solved. Low performance of the different technologies was also seen as severe, but solutions were considered even more likely to be found. The dominant concern of Japanese experts was cost, suggesting that technical solutions have largely been found to fuel cell problems, and the primary focus is now on commercialisation, and hence cost reduction.

Manufacturability and deployment barely register as issues, perhaps due to the inherent strength of the Japanese industrial players involved, and the very strong support of government. The latter was viewed as very positive by experts.

Policy is viewed as generally strongly supportive and likely to continue to be so. The major problems identified in this area were with regard to codes and standards, particularly for high pressure gas handling. Because the legislation considers all high pressure gases equally and is designed for industrial purposes, it is considered that solutions for hydrogen fuelling must be considerably over-engineered. This applies to both high pressure gas storage cylinders, for which revisions are being considered, and fuelling equipment.

Supply chain issues are also apparent in Japan. Smaller companies with specific technologies are not able to develop the manufacturing needed to bring down costs without large orders from OEMs.

\subsubsection{North America}

North American research and development into all aspects of hydrogen provision, storage and fuel cell drivetrains is very advanced, and the perspective of the experts is somewhat different from Japan, for example. North American vehicles tend to be larger than Japanese, giving more opportunities for integration of bulky components, but also requiring more power and energy to compete with the traditional solutions.

Codes and standards at a local level are also less harmonised in North America than in Japan, leading to concerns that the introduction of fuel cell vehicles could be hindered.

In North America, concerns were varied, though Fig. 13 shows several issues ranked as severe. Although few experts raised it, the acceptance of high pressure gas tanks was considered to be potentially very severe. However, high stack costs were considered more severe and by more experts, though also more likely to be solved.

The least likely issue to be solved was public support. The majority of experts viewed 'public' (i.e. government) support as insufficient and unfocused, and likely to hinder fuel cell vehicle roll-out. Several experts commented that an unprecented level of sustained support would be required to ensure suitable coordination between vehicle and infrastructure deployment, local codes and standards, education and other issues to give the best chances of success. In particular, government support for infrastructure was seen to be low.

Most North American experts considered that outstanding issues would be solved within 5 or 10 years (Fig. 14). Hydrogen storage had the longest time to solution, with cost, storage density and acceptance all cited as 10 -year issues.

No issue stood out over the others following concatenation (Fig. 15). Deployment was ranked as hardest to solve, closely followed by cost. However, all ranked around 2, showing that the experts did not believe these issues to be showstoppers.

\subsubsection{Europe}

The majority of experts interviewed in Europe had expertise in fuel cell drivetrains, though several specialists in storage and production were also interviewed. Experts identified a small number

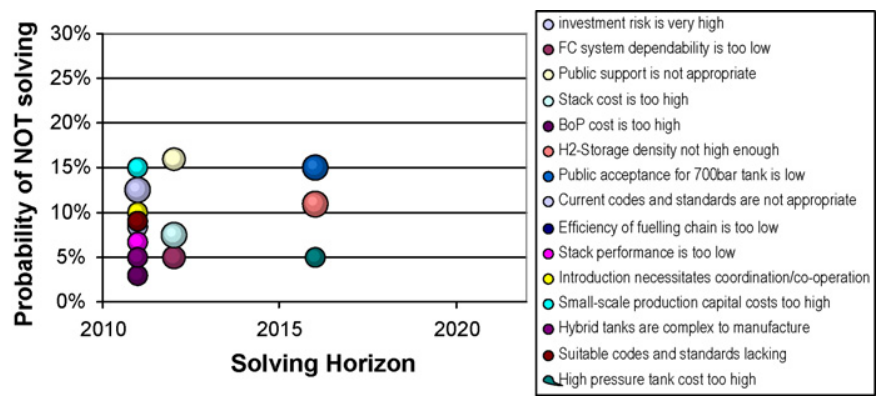

Fig. 14. Expert views on technology issues by solving horizon and improbability of solution (North America).

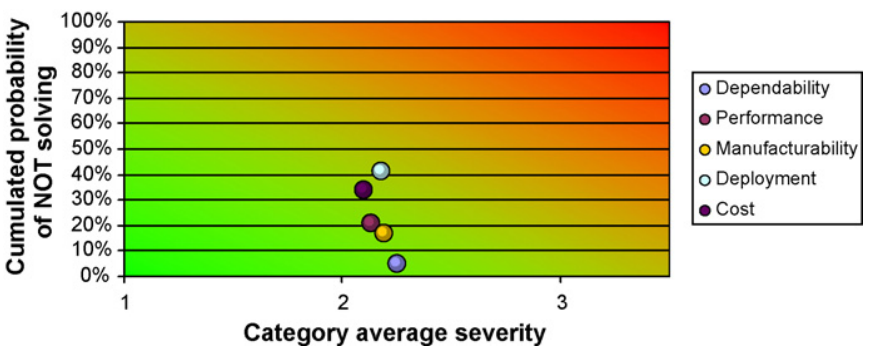

Fig. 15. Views on technology issues by severity and improbability of solution at issue category level (North America). 


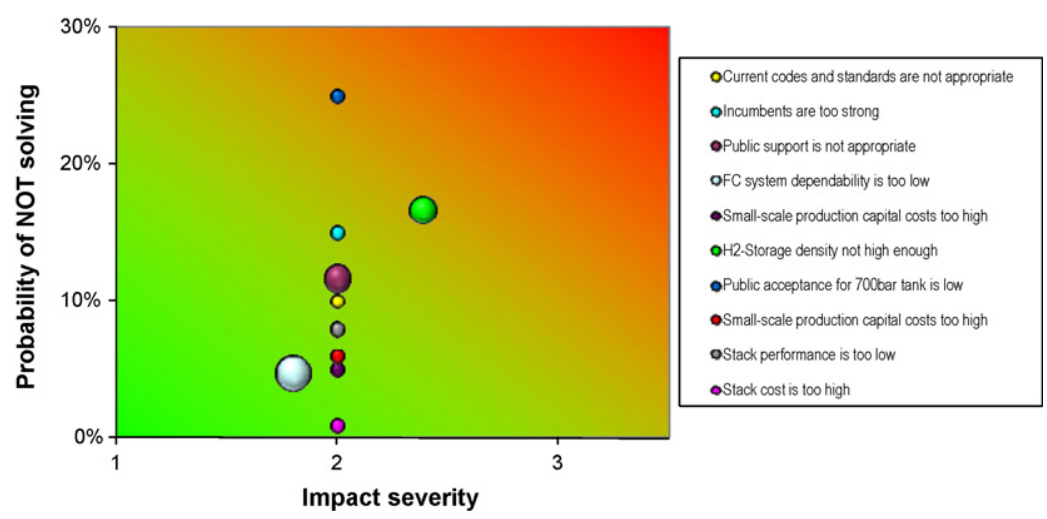

Fig. 16. Expert views on technology issues by severity and improbability of solution (Europe).

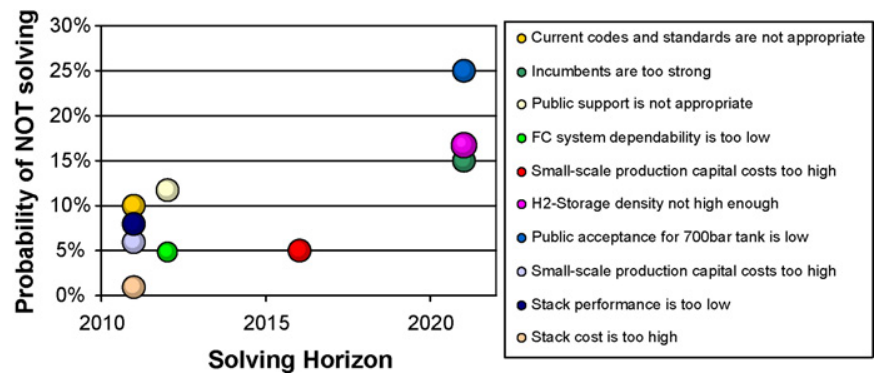

Fig. 17. Expert views on technology issues by solving horizon and improbability of solution (Europe).

of detailed technical issues requiring further work, in addition to a range of other, less important aspects.

Fig. 16 shows that few issues identified by European experts rank close to 3 in severity-only the issue of hydrogen storage density is still identified as a possible major problem. Intriguingly, however, this issue was ranked most highly by those not working directly on vehicles-OEMs and fuel cell drivetrain experts typically did not rank it as a severe issue. This suggests that perception may be the major issue. Public acceptance for 700 bar tanks is rated as having a low probability of solving, but only by one expert.

The most commonly identified issues related to fuel cell stack dependability, primarily lifetime. Most felt that the specific issues here, primarily operation in freezing conditions and insufficient MEA lifetime, would be solved in the future without technical breakthroughs.

Fig. 17 indicates that European experts were most cautious, estimating that related to hydrogen storage density, competing with strong incumbent technologies, and public acceptance for high pressure tanks would take around 15 years to solve. No technical issues related to fuel cell drivetrains were seen as further than 5 years from a probable solution.

Concatenating the different issues using the original categories allows us to show the primary concerns in Fig. 18. Deployment of fuel cell vehicle systems is clearly the category of most concern. Performance ranks similarly in both average severity and solving probability, but with fewer experts identifying that category. Cost is a potentially severe issue, but is strongly expected to be solved. Interestingly, manufacturability is not identified as a concern at all.

\section{Discussion}

Although some individual experts identified possible showstoppers, the overall position on technology issues was very positive, with many experts confident that pathways existed to solutions of specific problems, or that ways around them could be found. In gen-

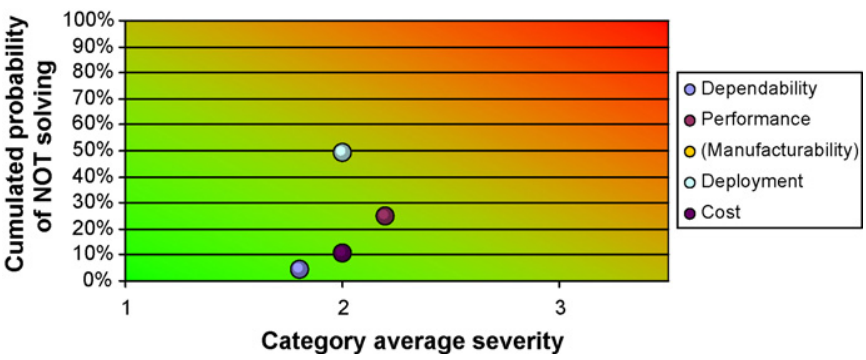

Fig. 18. Views on technology issues by severity and improbability of solution at issue category level (Europe). NB Manufacturability was not cited as an issue and hence is not shown above.

eral, it was believed that technical breakthroughs were not required for pre-commercial fuel cell vehicles to be on the road in tens of thousands within 5 years, and that full commercialisation could take place within 10-15 years. However, infrastructure, policy support, codes and standards and other 'soft' issues were considered to make this timeframe optimistic.

Perhaps surprisingly, hydrogen storage was not generally considered to be a showstopper. Although compressed tanks are not an optimal solution, it was agreed that they offer the only plausible pathway to commercialisation at this juncture, and that they will be the technology of choice for at least 10 , and probably 20 years. Experts agreed that they could work around the drawbacks of cost, high pressure gas handling and limited range by intelligent vehicle design, improved fuel cell system performance, and better packaging.

Regional differences emerged. Although experts outside China identified opportunities for China to leapfrog other regions and deploy fuel cell vehicles early, Chinese experts were less sanguine, citing the many other policy issues that need to be dealt with by government and the comparatively weak corporate support within China. Japan was identified by many experts as the prime location for sustained commercial deployment of fuel cell vehicles.

In Europe and North America, the majority of experts were positive about the prospects for fuel cell vehicle commercialisation. Several commented that the technology was less of an issue than the overarching deployment strategy, and that fuel cell cars would in any case be a late fuel cell market. Greater and more coherent government support was seen to be needed, not only in co-ordinating vehicle roll-out with infrastructure, but also in supporting supply chain development and in maintaining clear and long-term policies.

Building a suitable supply chain for automotive fuel cell parts was seen as extremely difficult, as existing suppliers typically do not understand fuel cells and fuel cell suppliers are not equipped to deal with producing hundreds of thousands of units at close to zero defect rates and low costs. It is not clear who should ensure that 
the investment in the supply chain will be made, i.e. who bears the risk, given that the bearer of the risk does not necessarily control it. Small-scale hydrogen production was widely viewed as a good starting point for infrastructure development.

The most potentially surprising conclusion from the interviews was that there are few, if any, technology showstoppers for the preliminary commercialisation of fuel cell vehicles, i.e. in tens of thousands. Some individuals did identify showstoppers, but the overall consensus was that technology would not be the main barrier. However, policy and deployment issues still require solutions.

\section{Acknowledgements}

We would like to thank Shell Hydrogen B.V. for its support throughout this study.

We would like to thank the following organisations for their invaluable contributions:

AIST (Japanese National Institute of Advanced Industrial Science and Technology)

- Tsukuba

- Osaka

- Tokyo

Argonne National Laboratory

DaimlerChrysler AG

Dalian Institute of Chemical Physics

Eon Consultants, Ltd.

Ford Motor Company

Fuyuan Century Fuel Cell

General Motors Corporation

Honda Motor Co., Inc.

Intelligent Energy

Interlink Corporation

International Council on Clean Transportation
JFE Container Co., Ltd.

Jiao Tong University, Shanghai

Johnson Matthey

Lawrence Livermore National Laboratory

Linde AG

Los Alamos National Laboratory

Ministry of Economy, Trade and Industry (Japan)

Mitsubishi Kakoshi Kaisha, Ltd.

Nissan Motor Co., Ltd.

NuCellSys

Osaka Gas Co., Ltd.

PolyFuel, Inc.

Questair, Inc.

Shanghai Municipal People's Government

Shanghai Shen-Li High Tech Co., Ltd.

Sunrise Power Co., Ltd.

Tokyo Gas Co., Ltd.

Tongji University, Shanghai

Toyota Motor Corporation

Tsinghua University, Beijing

UTC Power

ZSW (Zentrum für Sonnenenergie- und Wasserstoff-Forschung)

\section{References}

[1] R.K. Ahluwalia, X. Wang, Journal of Power Sources 177 (1) (2008) 167-176.

[2] F.R. Kalhammer, et al., Status and Prospects for Zero Emissions Vehicle Technology, Report of the ARB Independent Expert Panel 2007, Sacramento, CA, USA 2007, p. 207.

[3] W. Schmittinger, A. Vahidi, Journal of Power Sources 180 (2008) 1-14.

[4] J. Stumper, C. Stone, Journal of Power Sources 176 (2) (2008) 468-476.

[5] G. Landucci, A. Tugnoli, V. Cozzani, Inherent safety key performance indicators for hydrogen storage systems, Journal of Hazardous Materials, 159 (2-3) (2008) 554-566.

[6] M.P. de Wit, A.P.C. Faaij, International Journal of Hydrogen Energy 32 (18) (2007) 4859-4870. 